\title{
Intra-articular osteochondroma of the posteroinferior femoral neck associated with hip joint osteochondromatosis: A case report
}

\author{
SUNG IL WANG $^{1 *}$, EUN HAE PARK $^{2 *}$, SUN JUNG YOON $^{1}$ and JUNG RYUL KIM ${ }^{1}$ \\ Departments of ${ }^{1}$ Orthopaedics Surgery and ${ }^{2}$ Radiology, Chonbuk National University Medical School, Research \\ Institute for Endocrine Sciences and Research Institute of Clinical Medicine, Chonbuk National University-Biomedical \\ Research Institute of Chonbuk National University Hospital, Jeonju, Jeollabuk 561-756, Republic of Korea
}

Received June 28, 2017; Accepted September 4, 2017

DOI: $10.3892 / \mathrm{mco} .2017 .1411$

\begin{abstract}
Osteochondromas are usually extra-articular lesions originating from the metaphysis of long bones. Intra-articular osteochondromas may also occur, causing pain and discomfort and restricting the range of motion. Osteochondromas of the femoral neck are intra-articular lesions that are difficult to access for surgical resection, particularly when located posteriorly. We herein present a rare case of an intra-articular osteochondroma involving the posteroinferior aspect of the femoral neck associated with secondary synovial osteochondromatosis (SOC) of the hip joint in a 25-year-old woman. Determining the optimal treatment was difficult due to the high risk of avascular necrosis (AVN) following surgical excision. The patient was successfully treated with arthroscopic surgery and she remained in good condition at 2 years postoperatively, with a full range of motion of the hip joint, and without signs of limping, recurrence of the SOC, or AVN of the femoral head.
\end{abstract}

\section{Introduction}

Osteochondromas are usually extra-articular, as they predominantly originate from the metaphysis of long bones. However, intra-articular osteochondromas may also occur, causing pain and discomfort and restricting the range of motion (1). Osteochondroma of the femoral neck is rare. It has been described in patients with bursitis, sciatic nerve

Correspondence to: Dr Jung Ryul Kim, Department of Orthopaedics Surgery, Chonbuk National University Medical School, Research Insitute for Endocrine Sciences and Research Insitute of Clinical Medicine of Chonbuk National University-Biomedical Research Insitute of Chonbuk National University Hospital, 567 Baekje-ro, Dukjin-gu, Jeonju, Jeollabuk 561-756, Republic of Korea

E-mail: jrkeem@jbnu.ac.kr

${ }^{*}$ Contributed equally

Key words: osteochondroma, synovial osteochondromatosis, femoral neck, intra-articular joint compression, femoroacetabular impingement, and fracture at the stalk of the tumor (2-5). Determining the optimal treatment may be difficult due to the high risk of avascular necrosis (AVN) following surgical excision due to the lesion's location. We herein report a rare case of an intra-articular osteochondroma involving the posteroinferior aspect of the femoral neck associated with secondary synovial osteochondromatosis (SOC) of the hip joint in a 25-year-old woman, who was successfully treated with arthroscopic surgery.

\section{Case report}

A 25-year-old woman was referred to the Department of Orthopaedics Surgery of Chonbuk National University Hospital (Jeonju, Korea) for pain in the right inguinal region and a limping gait for 6 months. The pain was exacerbated upon standing and sitting. The patient had been receiving conservative treatment including rest, non-steroidal anti-inflammatory drugs and physical therapy at an outside hospital; however, the symptoms persisted. Physical examination of the right hip revealed a limited range of motion, with flexion $70^{\circ}$, extension $30^{\circ}$, external rotation $30^{\circ}$ and internal rotation $10^{\circ}$. The Patrick's test was positive. The results of the laboratory tests were within normal limits.

Radiographic examination revealed an osseous protuberance near the posteroinferior portion of the femoral neck. Oval-shaped ossifications were observed at the right hip joint (Fig. 1). There was no sign of fracture or pre-existing joint abnormality, such as osteoarthritis or osteonecrosis. Computed tomography was performed for precise evaluation of the numbers and locations of loose bodies. Loose bodies were relatively uniform in size with smooth margins, and some exhibited internal marrow trabeculation (Fig. 2). T1-weighted axial magnetic resonance imaging demonstrated an osseous protrusion at the posteroinferior aspect of the femoral neck, extending to the lesser trochanter. This osseous protuberance was connected to the bone marrow of the metaphysis, with a thin surrounding high-signal structure on fat-suppressed T2-weighted images (Fig. 3). These findings were indicative of cartilage. Multiple small intra-articular loose bodies $<5 \mathrm{~mm}$ in short diameter exhibited a low signal on both T1- and T2-weighted images. 
An arthroscopic approach was selected to minimize the potential for surgical morbidity. The patient was positioned supine on the fracture table with the affected limb in traction. First, the anterolateral portal was positioned over the superior and anterior borders of the great trochanter. A $70^{\circ}$-angled arthroscope was used for viewing the hip joint, and the anterior and posterolateral portals were viewed under direct vision. Saline was infused under a pressure of $60 \mathrm{mmHg}$ to assist with capsular distension during surgery. Several ossified loose bodies and synovitis of the capsule were observed (Fig. 4A). The osseous loose bodies of the acetabular fossa and surrounding space were removed (Fig. 4B). No lesions suggested osteochondral fracture or osteochondritis dissecans in the hip joint. Histologically, the loose bodies were round cartilaginous nodules with endochondral ossification. A central osteocartilaginous core was enclosed within fibrotic synovial tissue (Fig. 3C). The patient was discharged after postoperative wound care and was followed up for 2 years; at the last follow-up visit (August 2016) she remained in good condition, with a full range of motion of the hip joint and without signs of limping, SOC recurrence, or femoral head AVN (Fig. 5).

\section{Discussion}

Arthrotomy and arthroscopic surgery were considered for the treatment of the patient in the present case. Accessing posterior lesions of the femoral neck is difficult through the anterior surgical hip dislocation procedure reported by Ganz et al (6) The lesion is easily approached through a posterior dislocation procedure; however, the risk of soft tissue and vascular damage is high with this approach. The most worrisome consideration for surgical hip dislocation is the occurrence of postoperative femoral head AVN. As the median circumflex artery was close to the femoral neck lesion in our patient, she was considered to be at high risk for AVN of the femoral head associated with damage to the median circumflex artery.

To avoid femoral head AVN, arthroscopic removal of loose bodies of the acetabular fossa and surrounding space was performed. However, the limited working area in the hip joint complicated visualization and instrument manipulation. The anterior, anterolateral and posterolateral portals were used to address the limited visualization and working space. Visualization was improved by using a $70^{\circ}$ arthroscope, sufficient traction, and capsular distension of the hip joint.

Ilizaliturri et al (7) reported that the lateral synovial fold is a constant, reliable landmark for arthroscopically identifying the blood supply of the hip. Since the branches of the median circumflex artery are posterior to this landmark, capsular or bony resection must not be performed posterior to the lateral synovial fold. Therefore, a partial synovectomy was also performed in a limited area that did not exceed the lateral synovial fold. The patient has been followed up for 2 years, and she continues to have full range of motion of the hip joint, with no signs of limping, SOC recurrence, or femoral head AVN. However, the future possibility of osteochondromatosis recurrence and resection of the femoral neck osseous mass must be considered.

Multiple osseous loose bodies in the hip joint are usually caused by osteochondral fractures, osteochondritis dissecans, or pre-existing joint abnormalities, such as osteoarthritis,

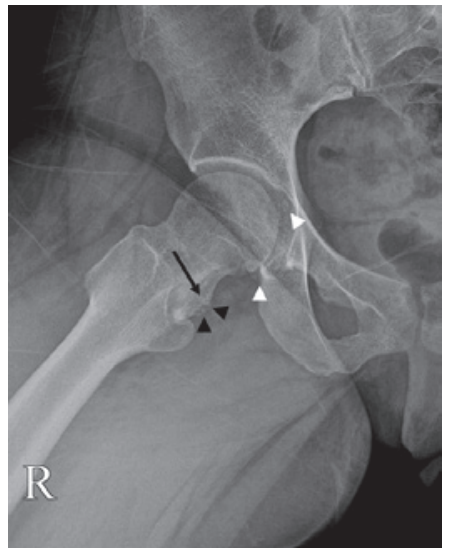

Figure 1. Radiograph of a 25-year-old woman presenting with pain in the right inguinal region. Radiographic frog leg view showing a protruding osseous mass at the posteroinferior aspect of the femoral neck (black arrow). Note the relatively similar sized oval osseous bodies around the lesion (black arrowheads) and hip joint (white arrowheads).

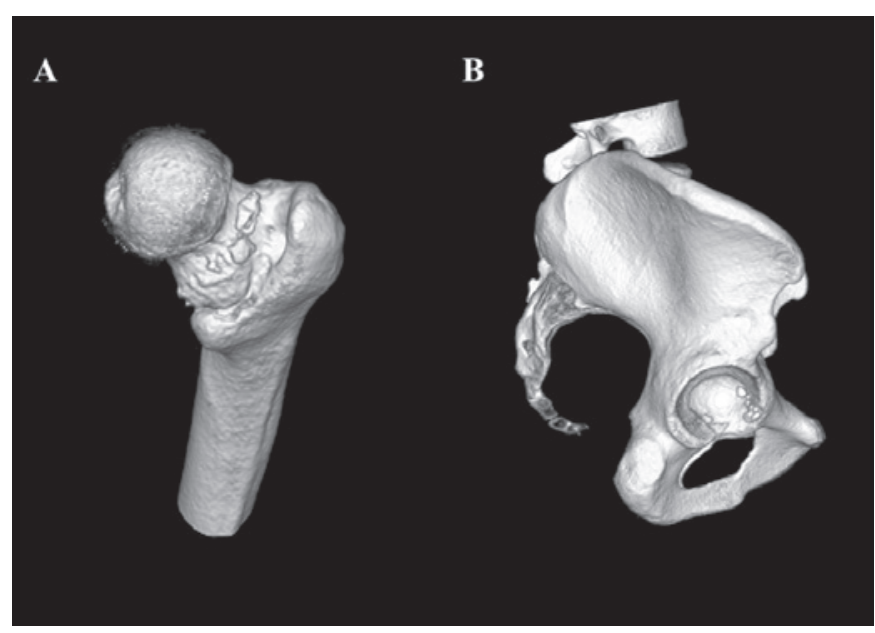

Figure 2. Computed tomography scan of the osseous mass in the right hip joint. (A) Three-dimensional reconstructed image of a bony protuberance at the posteroinferior aspect of the femoral neck and surrounding loose bodies. (B) Three-dimensional reconstructed image of the acetabulum with femur removal, demonstrating loose bodies within the acetabular cavity.

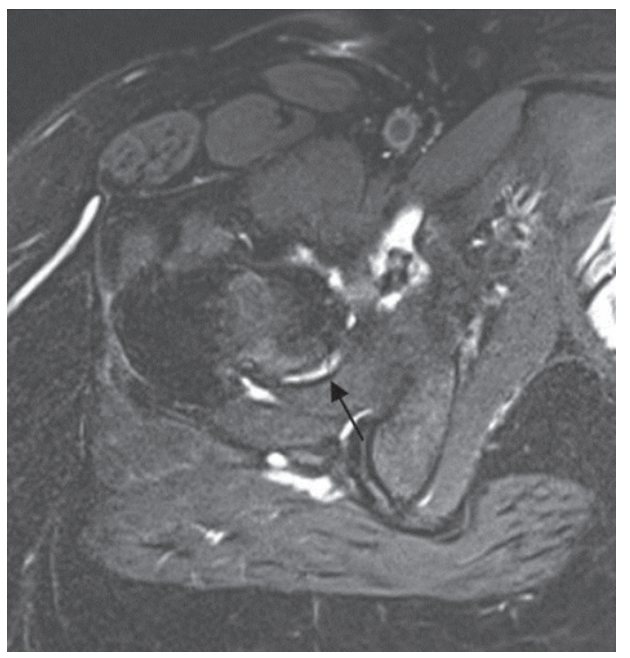

Figure 3. Axial fast spin echo fat-suppressed T2-weighted magnetic resonance image showing a mildly undulating high-intensity structure (arrow), compatible with a cartilaginous cap surrounding a bony excrescence. 

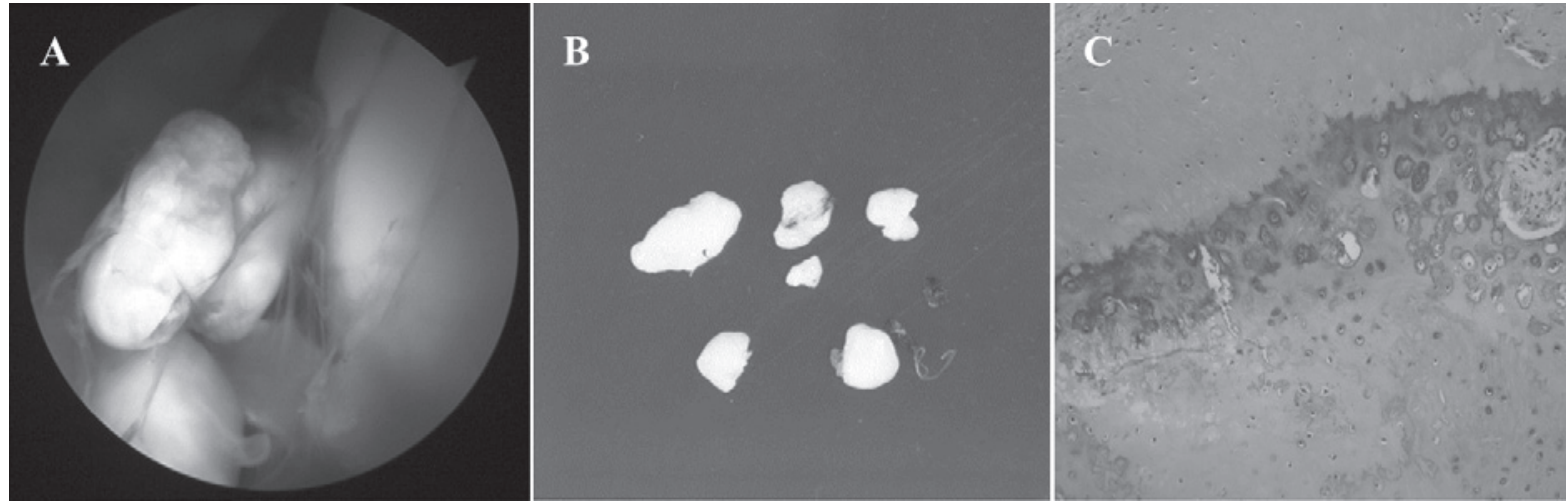

Figure 4. Arthroscopic and histological findings of loose bodies in the hip joint. (A) Arthroscopic image of loose bodies moving freely within the hip joint. (B) Cartilaginous loose bodies removed from the hip joint. (C) Hematoxylin and eosin-stained sections revealed an osteocartilaginous nodule encircled by fibrous synovial tissues (magnification, x200).

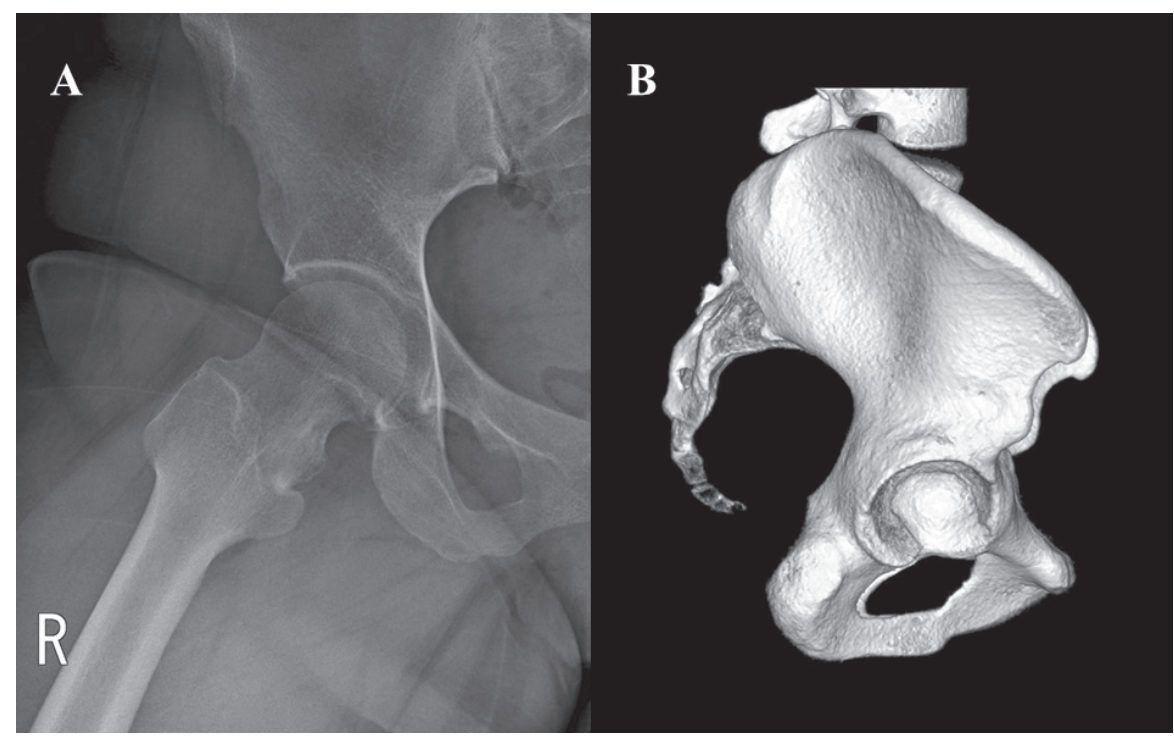

Figure 5. (A) Plain radiography and (B) computed tomography images obtained 2 years after arthroscopic treatment, revealing no loose bodies in the hip joint and no evidence of recurrence.

osteonecrosis, or rheumatoid arthritis. SOC may also be a rare cause. In present case, no pre-existing joint abnormalities, such as osteoarthritis, osteonecrosis, or rheumatoid arthritis were found. In addition, no lesions that suggested osteochondral fracture or osteochondritis dissecans were observed in the hip joint. Primary SOC was ruled out based on the absence of pathognomonic findings, such as numerous and relatively small osteochondral loose bodies throughout the joint (8).

Milgram et al hypothesized that osteochondral loose bodies may slowly grow with nourishment from the synovial fluid, while loose bodies are free of synovial attachments (9). Peh et al have described patients with secondary SOC around the osteochondromas of the femoral neck and pubic bone. They suggested that cartilaginous debris from the osteochondroma can stimulate metaplastic changes in the synovial lining, contributing to further development of osteochondral nodules (4).

In the present case, the presence of osteochondroma was considered to have played a significant role in the development of SOC. Due to the specific anatomy, mechanical blockage may occur through direct contact of the widened and enlarged femoral neck against the acetabular rim. This mechanism damages the labrum and adjacent articular cartilage. Although no definitive association between trauma and the development of SOC has been confirmed, limited evidence supports microtrauma as a precipitating factor in certain cases (10). The cartilaginous debris may have been nourished by synovial fluid to provoke repetitive traumatic inflammatory reactions and irritation caused by the loose body itself.

In conclusion, the appropriate treatment of an intra-articular osteochondroma involving the posteroinferior aspect of the femoral neck associated with secondary SOC may be difficult due to a high risk of AVN following surgical excision. The present case was successfully treated with arthroscopic removal of SOC, and the patient's symptoms completely resolved without recurrence.

The patient signed an informed consent for the publication of this case report and any accompanying images. Ethical approval of this study was waived by the Ethics Committee of Chonbuk National University Hospital, as this was a case report and the number of patients was $<3$. 


\section{References}

1. Siebenrock KA and Ganz R: Osteochondroma of the femoral neck. Clin Orthop Relat Res: 211-218, 2002.

2. Jung HT, Hwang DS, Jeon YS and Kim PS: Arthroscopic resection of osteochondroma of hip joint associated with internal snapping: A case report. Hip Pelvis 27: 43-48, 2015.

3. Kanauchi T, Suganuma J, Kawasaki T, Mochizuki R, Inoue Y, Uchikawa S, Kitamura K and Honda A: Fracture of an osteochondroma of the femoral neck caused by impingement against the ischium. Orthopedics 35: e1438-e1441, 2012.

4. Peh WC, Shek TW, Davies AM, Wong JW and Chien EP: Osteochondroma and secondary synovial osteochondromatosis Skeletal Radiol 28: 169-174, 1999.

5. Turan Ilica A, Yasar E, Tuba Sanal H, Duran C and Guvenc I: Sciatic nerve compression due to femoral neck osteochondroma: MDCT and MR findings. Clin Rheumatol 27: 403-404, 2008.
6. Ganz R, Gill TJ, Gautier E, Ganz K, Krügel N and Berlemann U: Surgical dislocation of the adult hip a technique with full access to the femoral head and acetabulum without the risk of avascular necrosis. J Bone Joint Surg Br 83: 1119-1124, 2001.

7. Ilizaliturri VM Jr, Orozco-Rodriguez L, Acosta-Rodríguez E and Camacho-Galindo J: Arthroscopic treatment of cam-type femoroacetabular impingement: Preliminary report at 2 years minimum follow-up. J Arthroplasty 23: 226-234, 2008.

8. Murphey MD, Vidal JA, Fanburg-Smith JC and Gajewski DA: Imaging of synovial chondromatosis with radiologic-pathologic correlation. Radiographics 27: 1465-1488, 2007.

9. Milgram JW: The development of loose bodies in human joints. Clin Orthop Relat Res: 292-303, 1977

10. Wright JM, Matayoshi E and Goldstein AP: Bursal osteochondromatosis overlying an osteochondroma of a rib. A case report. J Bone Joint Surg Am 79: 1085-1088, 1997. 